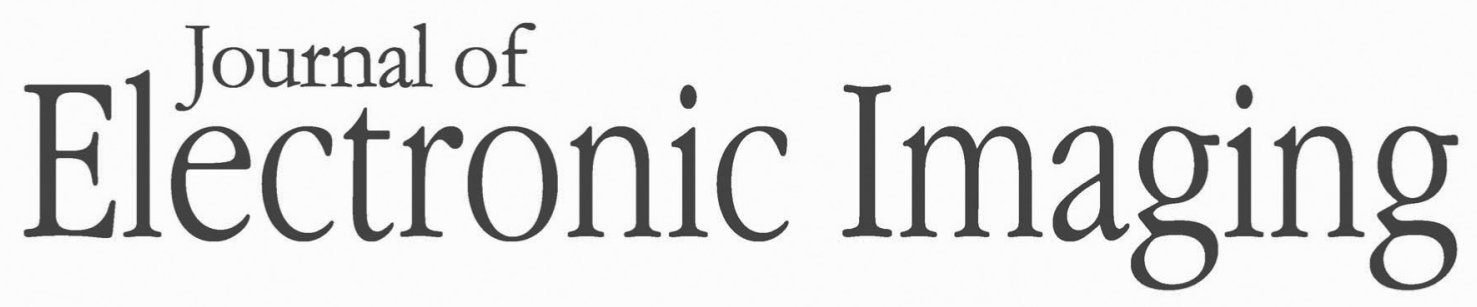

SPIEDigitalLibrary.org/jei

\title{
Errata: Haar-like compact local binary pattern for illumination-robust feature matching
}

Bongjoe Kim

Jihoon Choi

Sanghyun Joo

Kwanghoon Sohn

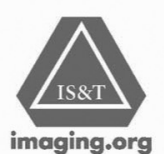




\title{
Errata: Haar-like compact local binary pattern for illumination-robust feature matching
}

\author{
Bongjoe Kim \\ Yonsei University \\ Department of Electrical and Electronic Engineering \\ 134 Shinchon-dong, Seodaemun-gu \\ Seoul 120-749, Republic of Korea \\ Jihoon Choi \\ Sanghyun Joo \\ Agency for Defense Development \\ Daejeon 305-152, Republic of Korea \\ Kwanghoon Sohn \\ Yonsei University \\ Department of Electrical and Electronic Engineering \\ 134 Shinchon-dong, Seodaemun-gu \\ Seoul 120-749, Republic of Korea \\ E-mail: khsohn@yonsei.ac.kr
}

[DOI: 10.1117/1.JEI.21.4.049801]

This article [J. Electron. Imaging 21(4), 043014 (2012)] was originally published online on 22 November 2012 with errors in two author affiliations.

Author affiliations of Sanghyun Joo and Kwanghoon Sohn were erroneously listed as Yonsei University, Department of Electrical and Electronic Engineering, 134
Shinchon-dong, Seodaemun-gu, Seoul 120-749, Republic of Korea. Both authors are actually affiliated with Agency for Defense Development, Daejeon 305-152, Republic of Korea, as listed above.

All online versions of the article were corrected on 11 December 2012. 